\title{
Comparison of some theoretical models for fittings of the temperature dependence of the fundamental energy gap in $\mathrm{GaAs}$
}

\author{
R. R. O. Morais, ${ }^{*}$ I. F. L. Dias, J. L. Duarte, and E. Laureto \\ Departamento de Física, Universidade Estadual de Londrina - UEL, CP6001, CEP 86051-970, Londrina, Paraná-Brazil \\ S. A. Lourenço \\ Instituto de Física, Universidade Federal de Uberlândia, CP593, CEP 38400-902, Uberlândia, MG, Brazil
}

\author{
E. C. F. da Silva and A. A. Quivy \\ Instituto de Física, Universidade de São Paulo - IFUSP, \\ CP66318, CEP 05315-970, São Paulo, São Paulo, Brazil
}

(Received on 24 November, 2008)

\begin{abstract}
In this work we report on a comparison of some theoretical models usually used to fit the dependence on temperature of the fundamental energy gap of semiconductor materials. We used in our investigations the theoretical models of Viña, Pässler- $p$ and Pässler- $\rho$ to fit several sets of experimental data, available in the literature for the energy gap of GaAs in the temperature range from 12 to $974 \mathrm{~K}$. Performing several fittings for different values of the upper limit of the analyzed temperature range $\left(T_{\max }\right)$, we were able to follow in a systematic way the evolution of the fitting parameters up to the limit of high temperatures and make a comparison between the zero-point values obtained from the different models by extrapolating the linear dependence of the gaps at high $T$ to $T=0 \mathrm{~K}$ and that determined by the dependence of the gap on isotope mass. Using experimental data measured by absorption spectroscopy, we observed the non-linear behavior of $E_{g}(T)$ of $G a A s$ for $T>\Theta_{D}$.
\end{abstract}

Keywords: Non-linear behavior of $E_{g}(T)$; Zero-point energy; Temperature dependence of the gap; Gallium arsenide

\section{INTRODUCTION}

The dependence on temperature of the energy gap, $E_{g}(T)$, is a very important characteristic of semiconductor materials and heterostructures and of great scientific and technological interest. For a large number of semiconductor materials, the energy gap decreases with the increase of the temperature and shows different behaviors depending of the analyzed range of temperature. At low $\left(T<0.02 \Theta_{D}\right.$, where $\Theta_{D}$ is the Debye temperature) and intermediary temperatures $\left(T=\Theta_{D}\right)$ the decrease of $E_{g}(T)$ is non-linear. For high temperatures $(T>>$ $\Theta_{D}$ ) the energy gap decreases linearly with temperature [1-3]. The linear decrease of $E_{g}(T)$ occurs due to the contribution of two distinct mechanisms: the electron-phonon interaction and the thermal expansion of the lattice [4-7]. In general, the contribution of the thermal expansion for the change of $E_{g}(T)$ is small as compared to the contribution of the electron-phonon interaction $[7,8]$. By this reason, several models proposed in the literature just take into account the electron-phonon interaction as a first approximation to the behavior of the $E_{g}(T)$ [9]. In the case of GaAs, the electron-phonon interactions which lead to a decrease of the energy gap with the temperature are associated to the longitudinal-acoustic (LA) and the longitudinal-optical (LO) phonons, being the dominant contribution due to the LA phonons [9-11].

Among several models usually used to describe $E_{g}(T)$, we find in the literature the distinguished models of Viña [12] (1984), Pässler- $p$ [9] and Pässler- $\rho$ [13] (1997). Essentially, these models differ among themselves with respect to the behavior of $E_{g}(T)$ at low temperatures. The model of Viña [12] shows a plateau behavior for $T<50 \mathrm{~K}$, making clear the absence of any dependence of the energy gap with temperature.

*Electronic address: rml.ronan@gmail.com
The Pässler- $\rho$ [13] model shows a quadratic dependence of $E_{g}(T)$ [14]. The Pässler- $p$ model shows a $T^{p}$ [1] dependence for $T<0.02 \Theta_{D}$ with $p=4$. For several semiconductor materials $E_{g}(T)$ still shows a $T^{p}$-dependence with the increase of temperature, but for values of $p$ in the range from 2 up to 3.3. In the limit of high temperatures $\left(T>>\Theta_{D}\right)$, all the models describe a linear asymptotic behavior of the energy gap with temperature given by[13]:

$$
E_{g}(T)=E_{g}(0)-\alpha_{i}\left(T-\frac{\Theta_{i}}{2}\right)
$$

where $\left.\alpha_{i}=-d E_{g i}(T) / d T\right)_{T} \rightarrow \infty=\alpha_{B}, \alpha_{p}$ or $\alpha_{\rho} . \Theta_{i}=\Theta_{B}$, $\Theta_{p}$ or $\Theta_{\rho}$ are empirical parameters related to the effective energy of the phonons in the temperature scale $\left.\left(\Theta_{e f f}=\langle\varepsilon\rangle / k_{B}\right)\right)$ associated to the models of Viña, Pässler-p and Pässler- $\rho$ respectively.

Extrapolating the linear dependence of $E_{g}(T)$ from high temperatures back to $T=0 \mathrm{~K}$, it is possible to estimate the renormalization of the energy gap at zero temperature, i.e., the zero-point energy $\Delta E(0)$ defined as $\Delta E(0)=E(0)-$ $E_{g}(0)$, where the non-renormalized energy gap, $E(0)$, is the energy of the crystalline system with immobile atoms at theirs equilibrium positions, and $E_{g}(0)$ is the renormalized gap at $T=0 \mathrm{~K}$. For the different models, $\Delta E(0)$ can be written as:

$$
\Delta E(0)=\alpha_{i} \frac{\Theta_{i}}{2} \equiv a_{i}
$$

with $\alpha_{i}$ and $\Theta_{i}$ as defined in Eq. (1). It is worthwhile to comment that, according to D. Rönnow et al [15], the renormalization of the gap is highly dependent on the model used to fit the temperature dependence of the energy gap. Still according to the same authors, the value of $\Delta E(0)$ estimated from the temperature dependence of $E_{g}(T)$ should be used as a criterion for the applicability of the different proposed models [15]. Results from the literature show that the values of $\Delta E(0)$ 
for $G a A s$ and others semiconductor materials seem to be dependent on the upper limit of the range of temperature used to perform the fits [16].

Due to the possibility to produce semiconductor materials with precise isotopic composition, nowadays it is possible to analyze the material properties as a function of the variation of the isotopic masses. At low temperatures, the change of the energy gap with the isotopic mass is proportional to $M^{-1 / 2}$, where $M$ is the mean effective masses of the constituent atoms of the material [17]. In the high temperature range, the energy gap does not depend on $M$. For GaAs, due to its similarity with $G e$ and due to the fact that $A s$ has only one stable isotope, the effect of the substitution of the ${ }^{69} \mathrm{Ga}$ and ${ }^{71} \mathrm{Ga}$ isotopes on the energy gap can be described by [18]:

$$
\frac{\partial E_{g}}{\partial M_{G a}}=\frac{1}{2}\left(\frac{1}{2 M_{G e}}\right) \Delta E(0)
$$

where $M_{G e}=70$ amu and $\Delta E(0)=53 \mathrm{meV}$. N. Garro et al [19] obtained $\partial E_{g} / \partial M_{G a}=0.39(0.06) \mathrm{meV} / \mathrm{amu}$ for the direct energy gap of $\mathrm{GaAs}$ using reflectivity measurements at $10 \mathrm{~K}$, and $0.43 \mathrm{meV} / \mathrm{amu}$ performing calculations with the pseudopotential method. If we use the experimental value $\partial E_{g} / \partial M_{G a}=0.39 \mathrm{meV} / \mathrm{amu}$ in Eq. (3), a value of $\Delta E(0)=$ $110 \mathrm{meV}$ is obtained for GaAs. As a matter of fact, the values of $\Delta E(0)$ that are found in the literature for $G a A s$ from estimates of isotopic substitution, linear extrapolation, and theoretical calculations are quite different $[16,18,20]$. According to our understanding, one important factor that must be taken into account for the analysis of these contrasting results is the lack of systematic experimental date for $T \geq \Theta_{D}$, which hides a possible non-linear behavior of $E_{g}(T)$ in the high temperature range. With respect to the issue, we notice that several estimates of $\Delta E(0)$ obtained by extrapolation procedures of the $E_{g}(T)$ curve at $T=0 \mathrm{~K}$ are obtained with parameters determined from fits in small domains of temperature, with the upper limit of the temperature interval smaller than the Debye temperature [16]. According to Pässler [3], a precise description of $E_{g}(T)$ and a reliable extrapolation procedure from high to very low temperatures can only be obtained if the experimental data set is measured up to or higher than the Debye temperature.

However, the Debye temperature of several materials is relatively high (for instance, $\Theta_{D}(C)=1880 \mathrm{~K}[3], \Theta_{D}(A l N)=$ $1020 \mathrm{~K}$ and $\left.\Theta_{D}(G a N)=870 \mathrm{~K}[21]\right)$, and there is a lack of experimental data for several materials at temperatures around $\Theta_{D}$. Experimental data from Panish [22], Shen [23] and Lautenschlager [11], usually used in the literature to describe $E_{g}(T)$ at high temperatures [3,24], covers a wide temperature range but have a reduced number of data points with a large numerical dispersion, which does not allow us to infer about the non-linear behavior of $E_{g}(T)$. In a relatively old work, Bludau et al [25] analyzing the dependence on temperature of the indirect energy gap of $S i$ have mentioned that it seems that the predicted linearity of $E_{g}(T)$ is not a precise description of the behavior of energy gap at high temperatures. Cardona and Thewalt [16] also make remarks about the accuracy of the results deriving from perturbation theory that keeps only up to the second order terms of the atomic displacements (proportional to $\left\langle u^{2}\right\rangle$ ) in the Hamiltonians used to describe the electron-phonon interactions. At high temperatures $\left\langle u^{2}\right\rangle$ is proportional to $T$, but the inclusion of higher order terms which are not considered in the linear approximation could lead to larger values of the exponents of $T$. In this way, procedures based on the linear approximation together with extrapolation from high to low temperatures or only the high temperature behavior of $E_{g}(T)$ can lead to incorrect values of the physical quantities as $\Delta E(0)$ or erroneous calculations of the thermal expansion contribution and the electron-phonon interaction to the change of the energy gap with temperature.

In the present work we analyzed the applicability of different theoretical models to describe to dependence of the fundamental energy gap with temperature in the range from 12 up to $957 \mathrm{~K} / 974 \mathrm{~K}$. To analyze the region for temperature $T$ $>300 \mathrm{~K}$ we used experimental data from several researchers $[11,22,23,26,27]$. Performing several fittings for different values of the upper limit of the analyzed temperature range $\left(T_{\max }\right)$, we were able to follow in a systematic way the evolution of the fitting parameters up to the limit of high temperatures and discuss the origin of the contrasting results related to the renormalization of the zero-point energy. Moreover, based on the experimental data of Johnson et al [27] we were able to confirm evidences of non-linear behavior of the GaAs energy gap for $T>300 \mathrm{~K}$.

\section{THEORETICAL DETAILS}

In this section, we present a short description of the theoretical expressions used to perform the fits of $E_{g}(T)$ versus $T$. The expression proposed by Viña et al [12] is given by:

$$
E_{g}(T)=E_{B}-a_{B}\left[1+\frac{2}{\exp \left(\Theta_{B} / T\right)-1}\right]
$$

where $E_{g}(T=0)=E_{B}-a_{B}$ is the energy gap at $T=0 \mathrm{~K}$; $a_{B}$ represents the strength of the electron-phonon interaction; $\Theta_{B}=\hbar \omega / k_{B}$ is a characteristic temperature representing the effective mean energy of the phonons $\left(\Theta \equiv\langle\varepsilon\rangle / k_{B}\right)$ on the temperature scale, which coincides with the phonon temperature in the single oscillator model, but does not have any correspondence with any peak in the phonon spectra; $\alpha_{B}=2 a_{B} / \Theta_{B}$ is the slope of the curve $E_{g}(T)$ versus $T$ as $T \rightarrow \infty$, i.e., $\alpha_{B} \equiv-\left(d E_{g}(T) / d T\right)_{T \rightarrow \infty}$. The material-specific degree of phonon dispersion (dispersion coefficient), given by $\Delta=\left[\left(\left\langle\varepsilon^{2}>-<\varepsilon\right\rangle^{2}\right) /\langle\varepsilon\rangle^{2}\right]^{1 / 2}$, is zero [28] in this case.

The expression proposed by Pässler [9] in the $p$-type model is given by:

$$
E_{g}(T)=E_{g p}(0)-\frac{\alpha_{p} \Theta_{p}}{2}\left[\sqrt[p]{1+\left(\frac{2 T}{\Theta_{p}}\right)^{p}}-1\right]
$$

where $E_{g p}(0)$ is the energy gap at $T=0 \mathrm{~K} ; \alpha_{p}$ is the slope of $E_{g}(T)$ as $T \rightarrow \infty$ i.e., $\alpha_{p} \equiv\left(d E_{g}(T) / d T\right)_{T \rightarrow \infty} ; \Theta_{p}$ is an empirical parameter associated to the effective mean energy of the phonons in the temperature scale $\Theta=\langle\varepsilon\rangle / k_{B}$ and is given by the expression $\Theta=\Theta_{p}[1.152+0.145 \ln (p-1.7)]$, with a dispersion coefficient given by $\Delta=\left(p^{2}-1\right)^{-1 / 2}$, where $p$ is an exponent of the spectral function.

The other model used in our work is the $\rho$-type Pässler 
model [13]:

$$
\begin{aligned}
& E_{g}(T)=E_{g \rho}(0)-\frac{\alpha_{\rho} \Theta_{\rho}}{2} \\
& {\left[\frac{\rho}{2}\left(\sqrt[4]{1+\frac{\pi^{2}}{6}\left(\frac{4 T}{\Theta_{\rho}}\right)^{2}+\left(\frac{4 T}{\Theta_{\rho}}\right)^{4}}-1\right)\right.} \\
& \left.+(1-\rho)\left(\operatorname{coth}\left(\frac{\Theta_{\rho}}{2 T}\right)-1\right)\right]
\end{aligned}
$$

where $E_{g \rho}(0)=E_{g}(T=0)$ is the energy gap at $T=0 \mathrm{~K} ; \alpha_{\rho}$ is the slope of $E_{g}(T)$ as $T \rightarrow \infty, \alpha_{\rho} \equiv-\left(d E_{g}(T) / d T\right)_{T \rightarrow \infty}$; $\Theta_{\rho}$ is an empirical parameter related to the cutoff frequency $\omega_{0}\left(\Theta_{\rho}=\hbar \omega_{0} / k_{B}\right)$ and to the effective mean energy of the phonons in the temperature scale and obeys the relation $\Theta_{r}=\Theta_{\rho}(1-0.5 \rho)$, with a dispersion coefficient given by $\Delta=(2-\rho)^{-1}[\rho(4-3 \rho) / 3]^{1 / 2}$. The $\rho$-parameter controls the relative weight of each type of interaction: $\rho \rightarrow 1$ represents the prevalence of the acoustic-phonon interaction, and $\rho \rightarrow 0$ represents the prevalence of interaction with optical phonons.

In order to determine the fit parameters related to each theoretical model, we used a chi-squared procedure. The criterion used to obtain the best-fit parameters was calculated according to the expression:

$$
S^{2}=\left(\frac{1}{n-m}\right) \sum_{j=1}^{n}\left(E_{g j}^{\exp }-E_{g j}^{a d j}\right)^{2}
$$

where $E_{g j}^{\exp }\left(E_{g j}^{a d j}\right)$ indicates the experimental (adjusted) values of the energy gap of the ith-data point. The factor $(n-m)$ is the number of degrees of freedom left after fitting $n$ data points with $m$ adjustable parameters in the fitting function.

\section{EXPERIMENTAL DETAILS}

The experimental data of the temperature dependence of the energy gap used in the present work were redigitalized from the following published data obtained with different characterization techniques: photoluminescence data from Grilli et al [26], in the range from $12 \mathrm{~K}$ up to $280 \mathrm{~K} / 33$ data-points; Lautenschlager et al [11] (ellipsometry), from $\sim$ $297 \mathrm{~K}$ to $510 \mathrm{~K} / 9$ points; Shen et al [23] (absorption) from $\sim$ $300 \mathrm{~K}$ to $881 \mathrm{~K} / 6$ points; Panish et al [22] (absorption), from $294 \mathrm{~K}$ to 974 / 5 points, and Johnson et al [27] (absorption) from $330 \mathrm{~K}$ to $957 \mathrm{~K} / 23$ points. Details of sample preparation and experimental procedure can be found in the cited references.

In order to perform a systematic analysis of the experimental results, we grouped the experimental data in two groups: I) data from $12 \mathrm{~K}$ to $280 \mathrm{~K}$ from Grilli et al [26], data from 294 $\mathrm{K}$ to $974 \mathrm{~K}$ from Lautenschlager et al [11], Shen et al [23], and Panish et al [22]; II) data from $12 \mathrm{~K}$ to $280 \mathrm{~K}$ from Grilli et al [26] and data from $330 \mathrm{~K}$ to $957 \mathrm{~K}$ from Johnson et al [27]. The experimental data from group I for $T>294 \mathrm{~K}$ is a very consistent set of data which allowed us to perform accurate fittings for temperatures above the room temperature but with a large dispersion in that whole temperatures range (see Fig. 1). A still more consistent data set analyzed by us consists of the absorption data measured in the Urbach-tail region by Johnson et al [27] (group II). So, according to our strategy, for the high temperature region we have one set of data with 20 data-points (group I) and another set with 23 data-points (group II) with almost equal upper limit temperatures (974 $\mathrm{K} / 957 \mathrm{~K}$ ), which enable us to perform a systematic analysis of the applicability of the different models to describe $E_{g}(T)$. From these experimental data we were also able to compare the results obtained from different data sets and to check anomalous behaviors of the obtained fit parameters.

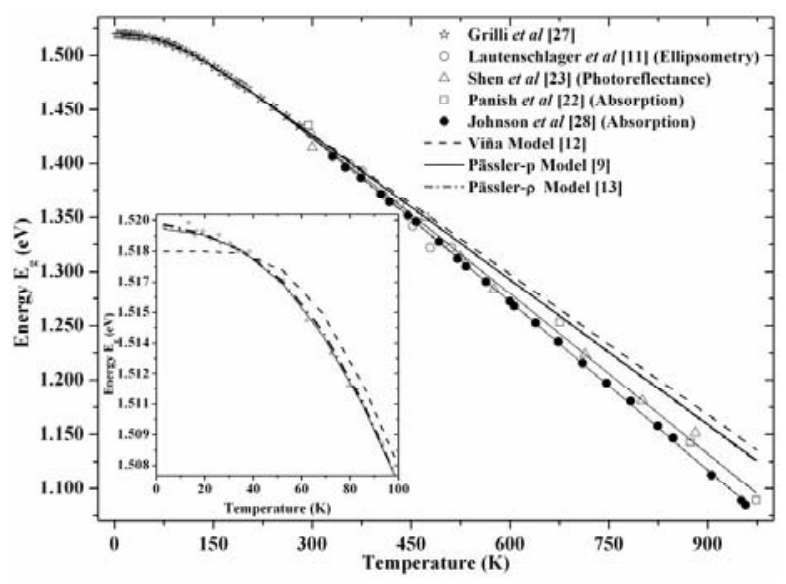

FIG. 1: Experimental data of $E_{g}(T)$ in the range from $\sim 294$ up to $\sim 974 \mathrm{~K}$ from Lautenchlager et al [11] (empty circle), Shen et al [23] (empty triangle), Panish et al [22] (empty square) and Johnson et al [27] (full circle) and experimental data from Grilli et al [26] in the range $12<T<280 \mathrm{~K}$. The inset shows the fitted curves to the experimental data from Grilli et al [26] (empty strar), at low temperatures, according to the Vinã, Pässler- $p$ and Pässler- $\rho$ models.

\section{RESULTS AND DISCUSSIONS}

In Fig. 1 we show the experimental data for $E_{g}(T)$ from the groups I and II in the temperature range $\sim 12<T<974(957)$ $\mathrm{K}$, and the fitted curves using the different models considered in the present work. The inset of this figure shows the redigitalized data of $E_{g}(T)$ in the range $\sim 12 \mathrm{~K}<T<\sim 100 \mathrm{~K}$ together with the fitted curves considering the whole analyzed domain of temperatures. We can observe in the region of low temperature the same pattern already observed in the literature $[13,29]$ with the Viña model showing a plateau behavior for $T=50 \mathrm{~K}$ and values of $S^{2}$ larger than that ones obtained with the other two models. The fit with the Pässler- $\rho$ model leads to an approximately quadratic behavior of $E_{g}(T)$ with a value of $S^{2}$ comparable to the one obtained with the Pässler- $\rho$ model. The values of the parameters obtained from the fits according to the different models are shown in Table I. The extrapolations to high temperatures $(T>280 \mathrm{~K})$ for $E_{g}(T)$ (see Fig. 1) and for the adjusted parameters (see Figs. 2-4) were performed using the data of Grilli et at [26] in the temperature range $12 \mathrm{~K}<T<280 \mathrm{~K}$. As can be observed, the curves obtained by extrapolation are located above the experimental points. This fact confirms that erroneous conclusions 
about the behavior of $E_{g}(T)$ at high $T$ are inferred if the experimental data are within a small temperature range. The fits obtained with the different models in the full temperature range coincide in the high temperature region for both groups of experimental data.

We see in Fig. 1 that the experimental values of $E_{g}(T)$ obtained by Johnson et al [27] are located, in general, below the data from group I. This fact is well evident when we observe the curves fitted to the different sets of experimental data. A possible explanation for this difference can be related to the different procedure used by Johnson et al [27] to obtain $E_{g}(T)$, which is based on observation of the Urbach tail. Another possibility which should also be considered is that the data of Johnson et al [27] were obtained with the same technique, the same procedure, the same sample in the whole domain of analyzed temperatures, which can provide a more systematic behavior for $E_{g}(T)$, with a smaller dispersion, differently from the others data sets.

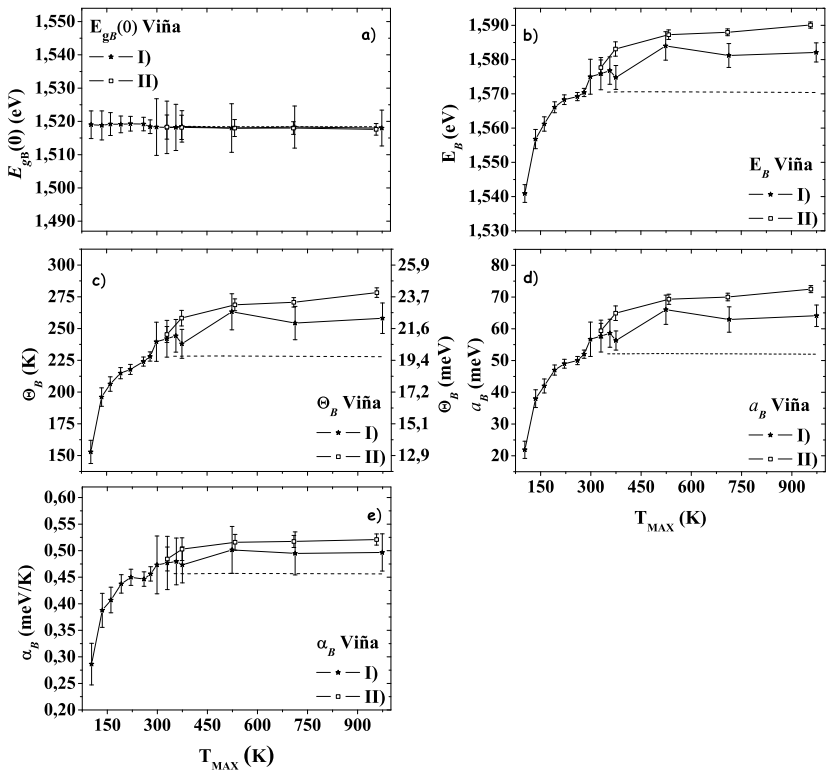

FIG. 2: Temperature range dependence of the fitting parameters obtained according to the Viña model [12] for the experimental data from group I (full star) and II (empty square). The dashed line is obtained by extrapolation of the parameters values determined from fits to the experimental data of Grilli et al [26]. The parameters $E_{g B}$ and $\alpha_{B}$ are calculated from the relations $E_{g B}=E_{B}-a_{B}$ and $\alpha_{B}=$ $2 a_{B} / \Theta_{B}$, respectively. The error bars are calculated by method of error propagation.

In Figs 2(b)-(d) we show the parameters $E_{B}, \Theta_{B}$, and $a_{B}$ obtained with the model of Viña [12]. In order to compare these data with the others obtained by us, we also show $E_{g B}(0)=E_{B}-a_{B}$ (Fig. 2(a)) and $\alpha_{B}=2 a_{B} / \Theta_{B}$ (Fig. 2(e)). The symbols represent the values of the parameters obtained in different ranges of temperature for different values of $T_{\max }$. The values of $E_{g B}(0)$, determined for different values of $T_{\max }$, are almost constant in the whole temperature range for the different sets of experimental data. The parameters $E_{B}, \Theta_{B}$, and $a_{B}$ obtained from group I increase in the range $12 \mathrm{~K}<T<$ $280 \mathrm{~K}$ showing a tendency to saturate for $T>350 \mathrm{~K}$. The parameters $E_{B}, \Theta_{B}$ and $a_{B}$ from group II show a slight tendency to increase with the increase of $T_{\max }$. The values for $\Delta E(0)$ obtained from the product $\alpha_{B}=2 a_{B} / \Theta_{B}$ using $T_{\max } \sim 3 \Theta_{D}$ $\left(\Theta_{D}=360 \mathrm{~K}\right.$ for $\left.\mathrm{GaAs}\right)$ are $\sim 64.1 \mathrm{meV}$ and $\sim 73 \mathrm{meV}$ for the two experimental data groups I and II respectively (see Table I). The values of the parameters determined from extrapolations to high temperatures stay constant with the increase of $T_{\max }$ and the obtained curves pass well below the experimental points. Therefore, we verify that to obtain consistent extrapolations using the Viña model it is necessary to have experimental data beyond the Debye temperature. The pa-
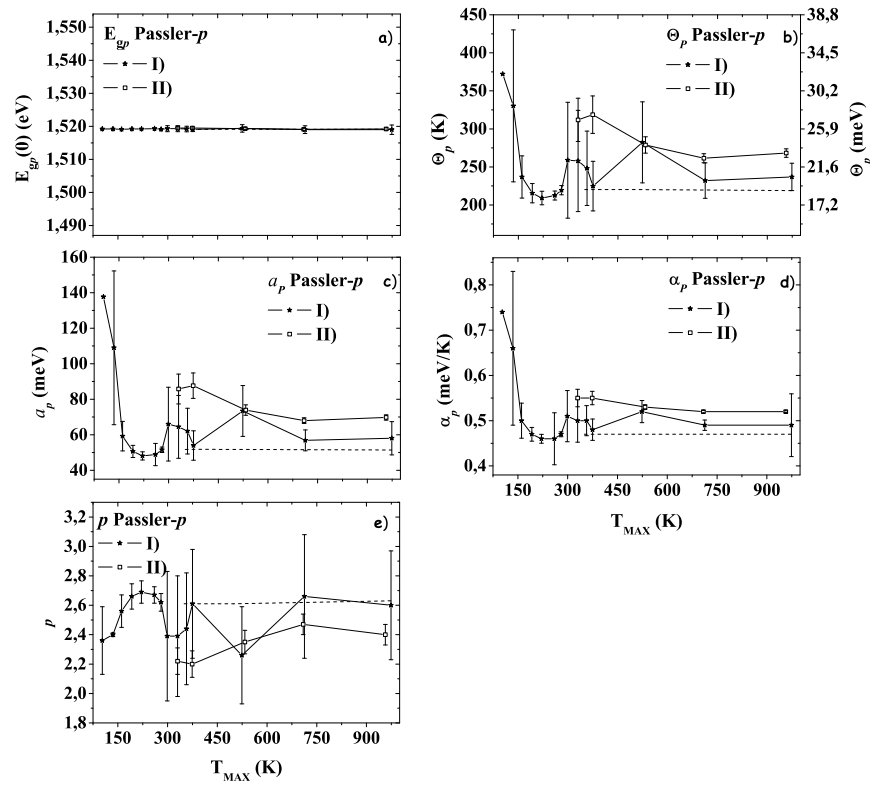

FIG. 3: Temperature range dependence of the fitting parameters obtained according to the Pässler- $p$ [9] for the experimental data from group I (full star) and II (empty square).The dashed line is obtained by extrapolation of the parameters values determined from fits to the experimental data of Grilli et al [26]. The parameter $a_{p}$ is calculated by $a_{p}=\alpha_{p} \Theta_{p} / 2$. The error bars are calculated by method of error propagation.

rameters obtained with the Pässler- $p$ model [9] are presented in Figs. 3(a)-(d). The parameter $a_{p}$ defined as $a_{p}=\alpha_{p} \Theta_{p} / 2$ is shown in Fig. 3(c). The values of the gap parameter $E_{g p}(0)$ (Fig. 3(a)) has practically a constant value with $T_{\max }$. The parameters $\Theta_{p}, \alpha_{p}$ and $a_{p}$ show a similar behavior with $T_{\max }$ (compare the shape of the curves in Figs. 3(b)-(d)). Concerning the results from group-II, we verified a step increase of $\Theta_{p}, \alpha_{p}$ and $a_{p}$ parameters in the range $280 \mathrm{~K}<T<350 \mathrm{~K}$ and a small decrease with further increase of the temperature. The parameter $p$ has values in the range from 2.4 up to 2.7 in the whole analyzed temperature range, which gives intermediary dispersion coefficients $(0.3<0.4(p=2.7)<\Delta<0.54$ $(p=2.4)<0.577)$ and leads to a small difference among the results from the different groups of data in the temperature range delimited by $T_{\max }$ (see Table I). In general, the standard behavior of the parameters determined from the different data sets by the Pässler- $p$ model is very similar. The curves obtained with the parameters determined from the extrapolation procedure are very close to the experimental data (see Fig. 3).

Based on our findings we can say that the extrapolations using the Pässler- $p$ model provide results more consistent with 
the experimental data for $T>280 \mathrm{~K}$. We also verified that the curves determined by extrapolation for $T>\Theta_{D}\left(\Theta_{D}=360 \mathrm{~K}\right.$ for $G a A s$ ) are superimposed to the experimental data in this temperature range. Larger errors in the range $280 \mathrm{~K}<T<$ $500 \mathrm{~K}$ are probably due to the dispersion of the group-I experimental data. The parameter $a_{p}$ tends to $\sim 58 \mathrm{meV}(\sim 68$ $\mathrm{meV}$ corrected) and to $70 \mathrm{meV}$ ( $84 \mathrm{meV}$ corrected) for the two experimental data group I and II respectively (see Table I).
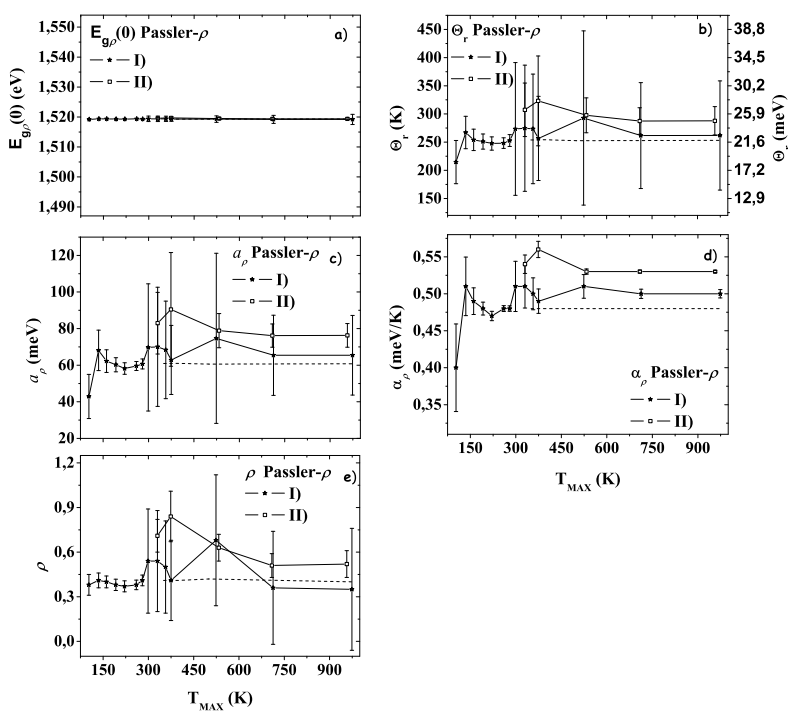

FIG. 4: Temperature range dependence of the fitting parameters obtained according to the Pässler- $\rho$ [13] for the experimental data from group I (full star) and II (empty square).The dashed line is obtained by extrapolation of the parameters values determined from fits to the experimental data of Grilli et al [26].The parameters $\Theta_{r}$ and $a_{\rho}$ are calculated by the equations $\Theta_{r}=\Theta_{\rho}(1-0.5 \rho)$ and $a_{\rho}=\alpha_{\rho} \Theta_{r} / 2$. The parameter $a_{p}$ is calculated by $a_{p}=\alpha_{p} \Theta_{p} / 2$. The error bars are calculated by method of error propagation.

In Figs. 4 (a)-(e) we show the parameters obtained from the Pässler- $\rho$ model [13]. We see in Fig. 4(a) that the values $E_{g \rho}(0)$ are practically constant with $T_{\max }$. The parameters $\Theta_{r}$, $a_{\rho}$ and $\alpha_{\rho}$ show similar trends with the increase of the temperature. The results of group II show an abrupt increase in the range $280 \mathrm{~K}<T<350 \mathrm{~K}$. The parameter $\Theta_{r}$ from group I show large error bars and oscillatory behavior around $500 \mathrm{~K}$. Moreover, the tendency of all the parameters with the increase of the temperature is practically the same and does not depend on $T_{\max }$. The $\rho$-parameter has values in the range from 0.4 up to 0.8 for groups I and II. However, its value is more concentrated in the region $0.3<\rho<0.6$, which leads to an intermediary dispersion in the range $0.328<\Delta<0.574$, in agreement with the criterion of applicability of the Pässler model [29]. Similarly to the results of the Pässler- $p$ model, the results obtained with Pässler- $\rho$ are also in excellent agreement with the experimental data and are more consistent when compared to the ones obtained with the Viña model. The parameter $a_{\rho}$ tends to $\sim 65 \mathrm{meV}$ and $\sim 76 \mathrm{meV}$ for the two experimental data group I and II respectively (see Table I). In general, the fitting parameters are strongly dependent of the range of temperature chose to perform the fits, i.e., the chosen value of $T_{\max }$, mainly in the range $12 \mathrm{~K}<T<450 \mathrm{~K}$ for the case of

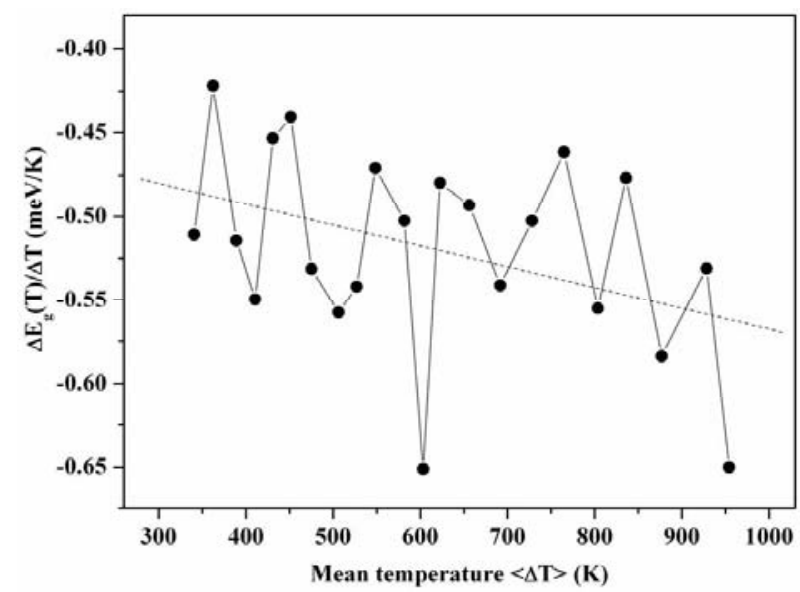

FIG. 5: Temperature dependence of the GaAs energy gap in the temperature range from $\sim 294$ up to $\sim 974 \mathrm{~K}$. The variation is calculated by the change of $E_{g}(T)$ at two consecutive temperatures divided by the respective temperature interval which that change occurred. The temperature mean value in each interval is plotted for each temperature interval.

the Viña model, and in the range $12 \mathrm{~K}<T<300 \mathrm{~K}$ for the Pässler- $p$ and Pässler- $\rho$ models. Therefore, the extrapolations of the fitted curves for $E_{g}(T)$ from the low temperature experimental data to the high temperature regions lead to parameters with very consistent values for $T>450 \mathrm{~K}$ for the Viña model, and $T>360 \mathrm{~K}$ for the Pässler- $p$ and Pässler- $\rho$ models. Considering $T_{\max }=974 \mathrm{~K}$ or $957 \mathrm{~K}$, the Pässler- $\rho$ model provides parameters with larger error bars. In order to correlate our findings with the $G a A s$ density of phonons we must take into account a correction for the value of $\Theta_{p}$ according to the expression $\Theta_{\text {eff }} \equiv<\varepsilon / k_{B}>=\Theta_{p}[1.152+0.145 \ln (p-1.7)]$ [3]. This correction increases about $10 \%$ to $20 \%$ the value of $\Theta_{p}$ if we consider the range $280 \mathrm{~K}<T<957 / 974 \mathrm{~K}$. Considering the fits performed for $T_{\max }(957 / 974 \mathrm{~K})$ the changes of the values of $k_{B} \Theta_{i}(\mathrm{i}=B, p$ effective, $\rho)$ due to the inclusion of the corrections vary from $22.2 \mathrm{meV}\left(\Theta_{B}\right.$ from group I) up to $27.8 \mathrm{meV}$ ( $\Theta_{\text {effp }}$ from group II), as can be seen in Table I. The values of $\Theta_{i}$ from the two data sets are, therefore, below the cut-off energy of the LA-phonon branch $\left(\varepsilon_{L A}=28 \mathrm{meV}\right)$. This result indicates that the decrease of the energy gap with temperature is mainly due to the interaction of electrons with LA phonons.

Complementary analysis, performed by expanding the range of temperature up to $T \sim 1179 \mathrm{~K}$ and the models of Viña and Pässler- $\rho$ along with the data from Beaudoin et al [30] which were obtained with a similar experimental procedure used by Johnson et al [27], show that the value of the $a_{i}$ parameter does not show an enlargement tendency with the increase the expansion of the temperature range, becoming stabilized around $\sim 75 \mathrm{meV}$ (Viña) and $\sim 79 \mathrm{meV}$ (Pässler$\rho)$ [31]. The values obtained here are therefore different from that founded in the literature obtained extrapolating the linear dependence of the gaps at high $T$ to $T=0 \mathrm{~K}$ and that determined by the dependence of the gap on isotope mass $[16,18,20]$. As we mention before, we consider that the lack of experimental data of $E_{g}(T)$ in the high- $T$ range in the fit models of $E_{g}(T)$ is one of the relevant factors to consider for 
Table I - Fitting parameters of $E_{g}(T)$ and their respective incertitudes according to Viña $\left(E_{g B}(0)\right.$, $\left.E_{B}, \Theta_{B}, a_{B}, \alpha_{B}\right)$ [12] , Pässler- $p\left(E_{g p}(0), \Theta_{p}, a_{p}, \alpha_{p}, p\right)$ [9] and Pässler- $\rho\left(E_{g \rho}(0), \Theta_{\mathrm{r}}, a_{\rho}, \alpha_{\rho}, \rho\right)$ [13] models for the temperature ranges $12 \mathrm{~K}<T<\sim 192 \mathrm{~K}, 12 \mathrm{~K}<T<280 \mathrm{~K}$, and $12 \mathrm{~K}<T<974 \mathrm{~K}$ for the group I experimental data and in the range $12 \mathrm{~K}<T<957 \mathrm{~K}$ for group II. The last column shows the values of $S^{2}$ (see Eq. 7).

\begin{tabular}{|c|c|c|c|c|c|c|c|}
\hline$\Delta T$ & $\begin{array}{l}E_{g B}(0) \\
(\mathrm{meV}) \\
\end{array}$ & $\begin{array}{c}E_{B} \\
(\mathrm{meV}) \\
\end{array}$ & \multicolumn{2}{|c|}{$\begin{array}{c}\Theta_{B} \\
(\mathrm{~K})(\mathrm{meV})\end{array}$} & $\begin{array}{c}a_{B} \\
(\mathrm{meV})\end{array}$ & $\begin{array}{c}\alpha_{B} \\
(\mathrm{meV} / \mathrm{K})\end{array}$ & $\begin{array}{c}S^{2} \\
\left(10^{-4} \mathrm{meV}^{2}\right)\end{array}$ \\
\hline I) $12-192$ & $1519.1 \pm 2.5$ & $1566.1 \pm 1.6$ & \multicolumn{2}{|c|}{$214.88(18.5) \pm 4.36$} & $47.0 \pm 1.6$ & $.43 \pm 1.73 \times 10^{-2}$ & $3.57 \times 10^{-4}$ \\
\hline I) $12-280$ & $1518.4 \pm 2.0$ & $1570.4 \pm 1.2$ & \multicolumn{2}{|c|}{$227.97(19.6) \pm 3.57$} & $52.0 \pm 1.3$ & $.46 \pm 1.35 \times 10^{-2}$ & $23.8 \times 10^{-4}$ \\
\hline I) $12-974$ & $1518.0 \pm 5.3$ & $1582.1 \pm 2.8$ & \multicolumn{2}{|c|}{$258.14(22.2) \pm 12.0$} & $64.1 \pm 3.4$ & $.50 \pm 3.50 \times 10^{-2}$ & $19.2 \times 10^{-2}$ \\
\hline II) $12-957$ & $1517.6 \pm 1.8$ & $1590.1 \pm 0.9$ & \multicolumn{2}{|c|}{$278.31(23.9) \pm 3.75$} & $72.5 \pm 1.1$ & $.52 \pm 1.05 \times 10^{-2}$ & $1.83 \times 10^{-2}$ \\
\hline \multicolumn{8}{|c|}{ b) Pässler-p } \\
\hline$\Delta T$ & $\begin{array}{l}E_{g p}(0) \\
(m e V)\end{array}$ & \multicolumn{2}{|c|}{$\begin{array}{c}\Theta_{p} \\
(K)(m e V)\end{array}$} & $\begin{array}{c}a_{p} \\
(m e V)\end{array}$ & $\begin{array}{c}\alpha_{p} \\
(\mathrm{me} V / K)\end{array}$ & $p$ & $\begin{array}{c}S^{2} \\
\left(10^{-4} \mathrm{meV}^{2}\right)\end{array}$ \\
\hline I) $12-192$ & $1519.2 \pm 0.07$ & \multirow{2}{*}{\multicolumn{2}{|c|}{$215.43(18.6) \pm 12.68$}} & $50.6 \pm 3.4$ & $0.47 \pm 0.0152$ & $2.66 \pm 0.086$ & $4.69 \times 10^{-4}$ \\
\hline I) $12-280$ & $1519.1 \pm 0.09$ & & & $51.6 \pm 5.72$ & $0.47 \pm 0.0054$ & $2.62 \pm 0.06$ & $5.17 \times 10^{-4}$ \\
\hline I) $12-974$ & $1519.0 \pm 1.42$ & \multicolumn{2}{|c|}{$236.90(20.4) \pm 18.0$} & $58.1 \pm 9.01$ & $0.49 \pm 0.0069$ & $2.6 \pm 0.37$ & $19.2 \times 10^{-2}$ \\
\hline II) $12-957$ & $1519.2 \pm 0.34$ & \multicolumn{2}{|c|}{$268.25(23.1) \pm 5.63$} & $69.7 \pm 1.50$ & $0.52 \pm 0.00169$ & $2.40 \pm 0.07$ & $1.07 \times 10^{-2}$ \\
\hline$\Delta T$ & & \multicolumn{2}{|c|}{$\begin{array}{c}\Theta^{e f f} p_{p} \\
(\mathrm{~K})(\mathrm{meV})\end{array}$} & $\begin{array}{c}a_{p f f}^{e f f} \\
(\mathrm{meV})\end{array}$ & & & \\
\hline I) $12-192$ & & \multicolumn{2}{|c|}{$249.45(21.5) \pm 14.53$} & $58.6 \pm 3.9$ & & & \\
\hline I) $12-280$ & & \multicolumn{2}{|c|}{$255.45(22.2) \pm 6.82$} & $60.0 \pm 1.80$ & & & \\
\hline I) $12-974$ & & \multirow{2}{*}{\multicolumn{2}{|c|}{$\begin{array}{l}276.53(23.8) \pm 20.55 \\
322.90(27.8) \pm 6.27\end{array}$}} & $67.8 \pm 10.8$ & & & \\
\hline II) $12-957$ & & & & $84.0 \pm 1.7$ & & & \\
\hline \multicolumn{8}{|c|}{ c) Pässler- $\rho$} \\
\hline$\Delta T$ & $\begin{array}{l}E_{g \rho}(0) \\
(\mathrm{meV})\end{array}$ & \multicolumn{2}{|c|}{$\begin{array}{c}\Theta_{r} \\
(K)(m e V)\end{array}$} & $\begin{array}{c}a_{\rho} \\
(\mathrm{meV})\end{array}$ & $\begin{array}{c}\alpha_{\rho} \\
(\mathrm{meV} / \mathrm{K})\end{array}$ & $\rho$ & $\begin{array}{c}S^{2} \\
\left(10^{-4} \mathrm{meV}^{2}\right)\end{array}$ \\
\hline I) $12-192$ & $1519.3 \pm 0.07$ & \multicolumn{2}{|c|}{$251.01(21.6) \pm 13.49$} & $60.2 \pm 3.8$ & $0.48 \pm 0.087$ & $0.38 \pm 0.04$ & $4.61 \times 10^{-4}$ \\
\hline I) $12-280$ & $1519.3 \pm 0.09$ & \multicolumn{2}{|c|}{$252.80(21.8) \pm 10.5$} & $60.7 \pm 2.72$ & $0.48 \pm 0.004$ & $0.41 \pm 0.04$ & $4.84 \times 10^{-4}$ \\
\hline I) $12-974$ & $1519.2 \pm 1.70$ & \multicolumn{2}{|c|}{$261.80(22.6) \pm 96.8$} & $65.4 \pm 21.8$ & $0.50 \pm 0.01$ & $0.35 \pm 0.41$ & $19.3 \times 10^{-2}$ \\
\hline II) $12-957$ & $1519.4 \pm 0.30$ & \multicolumn{2}{|c|}{$287.90(24.8) \pm 25.2$} & $76.1 \pm 6.5$ & $0.53 \pm 0.001$ & $0.52 \pm 0.08$ & $1.01 \times 10^{-2}$ \\
\hline
\end{tabular}

the precise description of $E_{g}(T)$. In face of the dispersion of results for $\Delta E(0)$ values from both procedures (extrapolating the linear dependence of the gaps at high $T$ to $T=0 \mathrm{~K}$ and the dependence of the gap on isotope mass) we inferred that or the linear dependence of $E_{g}(T)$ at high temperatures ( $T$ $\left.>>\Theta_{D}\right)$ is not reached in the analyzed temperature range or, as pointed out by Cardona and Thewalt [16], electron-phonon anharmonic effects lead to a higher-order dependence of the energy gap with temperature. Therefore, the choice of upper limit of temperature to which $E_{g}(T)$ has a linear dependence with $T$ is arbitrary, and can lead to incorrect values of $\Delta E(0)$ when Eq. 2 is used.

For instance, using the Pässler- $p$ model [9] to describe the temperature dependence of the energy gap of $\mathrm{GaAs}$ in the range from $2 \mathrm{~K}$ to $280 \mathrm{~K}$, and the values $\Theta_{p}=226 \mathrm{~K}, \alpha_{p}$ $=0.347 \mathrm{meV} / \mathrm{K}$ and $p=2.51$, Cardona et al [18] estimated $\Delta E(0)=53 \mathrm{meV}(-53 \mathrm{meV}$ in the Cardona [16] notation) from the photoluminescence data measured by Grilli et al [26]. Using another set of experimental data with a different upper limit defining the domain of temperature $(974 \mathrm{~K} \sim$ $\left.2.7 \Theta_{D}\right)$, Cardona et al $[18]$ obtained $\Delta E(0)=90 \mathrm{meV}$. As can be find out from these results, the values of $\Delta E(0)$ obtained for $G a A s$ seem to be dependent on the upper limit of the domain of temperature used to perform the fits. It is also important to say that the value of $90 \mathrm{meV}$ was obtained from the fits of two sets of experimental results: (i) the data obtained by Grilli et al [26] in the range $2 \mathrm{~K}<T<280 \mathrm{~K}$ and (ii) the absorption data obtained by Panish et al [22] in the high temperature region $(294 \mathrm{~K}<T<974 \mathrm{~K})$. However, these data for high temperatures were recorded in a relatively large tem- perature range with a reduced set of data points (5 points), which prejudices the fits and the carried out analysis.

The lack of systematic experimental data for temperatures above the room temperature is an entanglement for the analysis of $E_{g}(T)$ for a large number of materials. The data from Johnson et al [27] allow, a more systematic analysis of $E_{g}(T)$ for $G a A s$. Using the finite difference method to analyze the experimental data of $E_{g}(T)$ obtained by Johnson et al [27], it is possible to visualize the change of the parameter $\alpha_{\text {exp }}=-\Delta E_{\text {gexp }}(T) / \Delta T$ by performing derivatives in successive ranges of $E_{g}(T)$. In Fig. 5 we show the values of $\alpha_{\exp }$ as a function of the mean temperature for each interval $\Delta T$ obtained with the data of Johnson et al [27]. Therefore, $\alpha_{\text {expn }}$ is given by:

$$
\alpha_{\exp n}=\frac{\Delta E_{g}(T)}{\Delta T}=\frac{E_{g}\left(T_{n}\right)-E_{g}\left(T_{n-1}\right)}{T_{n}-T_{n-1}}
$$

where $T_{n}$ and $T_{n-1}$ are consecutive values of $T$, and $E_{g}\left(T_{n}\right)$, $E_{g}\left(T_{n-1}\right)$ are the respective experimental values of the energy gap. In general, in the range from $330 \mathrm{~K}$ up to 957 $\mathrm{K}$, the value of $\alpha_{\text {expn }}$ decreases from -0.47 to $-0.55 \mathrm{meV} / \mathrm{K}$ between two consecutive intervals. This variation of $\alpha_{\text {expn }}$ is relatively large $(\sim 17 \%)$ considering the predicted linear behavior of $E_{g}(T)$ at high temperatures. The non-linear behavior of $E_{g}(T)$ was already foreseen by Bludau et al [25] who used second order polynomes to fit the temperature dependence of $S i$ indirect-energy gap. Cardona and Thewalt [16] also point out the importance of anharmonic terms (which leads to higher order terms in $T$ ) in the electron-phonon interaction to analyze the temperature dependence of the lattice 
parameter of Si. The experimental data of $E_{g}(T)$, from the results of Johnson et al [27], even in the limit of high temperatures, show a small curvature that must be taken into account when we analyze the temperature dependence of the energy gaps. The extrapolation from the linear dependence at high temperature to low temperature tends to be done with some degree of arbitrariness, which can explain the contrasting results of $\Delta E(0)$ obtained with from the different experimental procedures. Therefore, the results of Johnson et al [27] allowed us confirm the non-linear temperature dependence of the GaAs energy gap at high temperature. So, our findings indicate that more systematic experimental studies searching the high temperature region $\left(T>>\Theta_{D}\right)$ must be performed in order to obtain a precise description of the dependence of the energy gap with temperature.

\section{CONCLUSIONS}

In this work we analyzed the temperature dependence of the GaAs energy gap using different sets of experimental data. We performed fits of $E_{g}(T)$ versus $T$ in different domains of temperature defined by different upper temperatures. We were able to make a systematic comparison of the fit- ting parameters obtained with three theoretical models in a large temperature range ( $12 \mathrm{~K}$ to $974 \mathrm{~K} / 957 \mathrm{~K})$. The values obtained for the zero point renormalizations energy $-\Delta E(0)$ - from different experimental data groups and from different theoretical models for fitting the temperature dependence of $G a A s$ obtained extrapolating the linear dependence of the gaps at high $T$ to $T=0 \mathrm{~K}$ varying between $64.1 \mathrm{meV}$ (Viña model) and $84 \mathrm{meV}$ (Pässler-p model).

Using absorption experimental data measured in temperatures higher than the room temperature we were able to observe the non-linearity of $E_{g}(T)$ as already reported in the literature, with a quite large value for $d E_{g}(T) / d T$ in the range $280 \mathrm{~K}<T<957 \mathrm{~K}$. We believe that a more realistic description of $E_{g}(T)$ can only be obtained if the effects of the non-linearity at high temperatures are included in the theoretical models describing the dependence on temperature of the energy gap.

\section{ACKNOWLEDGEMENTS}

The authors would like to thank the financial support granted by the Brazilian agencies CAPES, CNPq, Fundação Araucária and FBB.
[1] M. Cardona, T. A. Meyer and M. L. W. Thewalt, Phys. Rev. Lett. 92, 196403-1 (2004);

[2] P. Debye, Ann. Phys. 22, 180 (1907);

[3] R. Pässler, Phys. Status Solidi (b) 216, 975 (1999);

[4] P. B. Allen and V. Heine, J. Phys. C: Solid State 9, 2305 (1976);

[5] S. Zollner, S. Gopalan and M. Cardona, Sol. Stat. Commun. 77, 485 (1945);

[6] P. B. Allen and M. Cardona, Phys. Rev. B 23, 1495 (1981);

[7] A. Manoogian and J. C. Wooley, Can. J. Phys. 62, 285 (1984);

[8] J. P. Walter, R. R. L. Zucca, M. L. Cohen and Y. L. Shen, Phys. Rev. Lett. 24, 102 (1970);

[9] R. Pässler, Phys. Status Solidi (b) 200, 155 (1997);

[10] C. Lárez and C. Rincón, J. Phys. Chem. Solids 58, 1111 (1997);

[11] P. Lautenschlager, M. Garriga, S. Logothetidis and M. Cardona, Phys. Rev. B 35, 9174 (1987);

[12] L. Viña, S. Logohtetidis and M. Cardona, Phys. Rev. B 30, 1979 (1984);

[13] R. Pässler, J. Appl. Phys. 83, 3356 (1998);

[14] R. Pässler, E. Griebl, H. Riepl, G. Lautner, S. Bauer, H. PÇreis, W. Gebdhardt, P. Buda, D.J. As, D. Schikora, K. Lischka, K. Papagelis, S. Ves, J. Appl. Phys. 86, 4403 (1999);

[15] D. Rönnow, L. F. Lastras-Matínez and M. Cardona, Eur. Phys. J. B 5, 29 (1998);

[16] M. Cardona and M. L. W. Thewalt, Rev. Mod. Phys. 77, 1173 (2005);
[17] C. Parks, A. K. Ramdas, S. Rodriguez, K. M. Itoh and E. E. Haller, Phys. Rev. B 49, 14244 (1994);

[18] M. Cardona, Phys. Status. Solid. (a) 188, 1209 (2001);

[19] N. Garro, A. Cantarero, M. Cardona, A. Göbel, T. Ruf. And K. Eberl, Phys. Rev. B 54(7), 4732 (1996);

[20] T. A. Meyer, D. Karaiskaj, M. L. W. Thewalt and M. Cardona, Sol. Stat. Commun. 126, 119 (2003);

[21] R. Pässler, J. Appl. Phys. 90, 3956 (2001);

[22] M. B. Panish and H. C. Casey, Jr., J. Appl. Phys. 40, 163 (1969);

[23] H. Shen, S. H. Pan, Z. Hang, Jin Leng, Fred. H. pollak, J. M. Woodall and R. N. Sacks, Appl. Phys. Lett. 53, 1080 (1988);

[24] C. D. Thurmond, J. Electrochem. Soc.:Solid State Science and Technology 122, 1134 (1975);

[25] W. Bludau, A. Onton and W. Heinke, J. Appl. Phys. 45, 1846 (1974);

[26] E. Grilli, M. Guzzi, R. Zamboni and L. Pavesi, Phys Rev. B 45, 1638 (1992);

[27] S. R. Johnson and T. Tiedje, J. Appl. Phys. 78, 5609 (1995);

[28] R. Pässler, J. Appl. Phys. 88, 2570 (2000);

[29] R. Pässler, Phys. Rev. B 66, 085201-1 (2002);

[30] M. Beaudoin, A. J. G. DeVries, S. R. Johnson, H. Laman and T. Tiedje, Appl. Phys. Lett. 70, 3540 (1997);

[31] R. R. O. Morais, to publish 